\title{
Synthesis and photophysical properties of fluorine substituted end group for benzo[1,2-b:4,5-b'] diselenophene-based small molecule acceptor
}

\author{
Yan-Qiang Liu ${ }^{1}$, Jin-Liang Wang ${ }^{1 *}$ \\ ${ }^{1}$ School of Chemistry and Chemical Engineering, Beijing Institute of Technology, Beijing 100081, China.
}

\begin{abstract}
A-D-A types non-fullerene small molecule acceptors (NF-SMAs) have achieved excellent progress in recent years, while the development of series of new materials. Herein, a new NF-SMA, named BDSe-4F, based on linear benzo[1,2-b:4,5-b'] diselenophene with the difluorinated 5,6-difluoro-3(dicyanomethylene) indanone (IC-2F) was synthesized and investigated. Due to the introduction of IC-2F, the intramolecular charge transfer is enhanced. Furtherly, the BDSe-4F exhibits a wider absorption from 600$800 \mathrm{~nm}$ in solution. From solution to film, the absorption spectrum shows an obvious red-shifted in the range of $800-900 \mathrm{~nm}$ and the maximum absorption peak red-shifted at $31 \mathrm{~nm}$, suggesting that much stronger intermolecular interactions caused by non-covalent bond of F...S, F...Se, and S...O. The BDSe-4F possesses good spectral absorption range and excellent molecular stacking, which beneficial to achieve high $J_{\mathrm{SC}}$ and electron mobility. These results illustrate that the synergistic strategy of using benzo[1,2-b:4,5-b'] diselenophene core unit and $\mathbf{I C - 2 F}$ end group is a promising strategy to enhance performance in organic solar cells.
\end{abstract}

\section{Introduction}

Solution-processed bulk-heterojunction (BHJ) organic solar cell (OSCs) have advantages of flexible, low-cost and large-area printing via roll-to-roll process.[1-4] The power conversion efficiencies (PCE) of OSCs could improve along with synthesis of new materials and innovation of fabrication techniques.[5-7] On the other hand, as the important component of the active layer, nonfullerene small molecule acceptors (NF-SMAs) play an important role in order to blend with SMAs scientifically and achieve optimal morphology. At present, the PCE have exceeded $17 \%$ for single junction cells, [8-11] indicating that OSCs have potential commercial application value.

Over the last few years, lots of molecular engineering strategies have been exploited in the synthesis of A-D-A type NF-SMAs to improve the performance of organic solar cells.[12-15] Generally, the design strategies of AD-A type NF-SMAs depend on the following two parts: the central donor core (D) and electron-withdrawing end groups (A). Thus, NF-SMAs could be easily regulated absorption spectrum, energy levels and film morphology by changing D or A unit.[16-17] Benzothiophene (BDT) and its derivatives possessed good plane nature and electron-rich feature has been widely used as D unit to structure rich electronic core.[18] In addition, introducing halogen atoms into the NF-SMAs is an effective strategy to regulate the lowest unoccupied molecular orbital (LUMO) and highest occupied molecular orbital (HOMO) energy levels simultaneously, such as introducing strong electronegativity units on donor core or using strong electron-withdrawing end groups.[19] 5,6-difluoro-3(dicyanomethylene)indanone (IC-2F), 5,6-dichloro-3(dicyanomethylene)indanone (IC-2Cl) and 5,6-bibromo3-(dicyanomethylene)indanone (IC-2Br) both can enhance intermolecular $\pi-\pi$ interactions and improve intermolecular charge transfer owing to the push-pull electronic effect of donor core and end groups.

Herein, we designed and synthesized a new NF-SMA BDSe-4F based on linear benzo[1,2-b:4,5- $\left.\mathrm{b}^{\prime}\right]$ diselenophene as donor core with the difluorinated 5,6difluoro-3-(dicyanomethylene) indanone (IC-2F) electron-withdrawing units. The BDSe-4F exhibits a narrow optical band gap of $1.45 \mathrm{eV}$. Due to strong electron-rich core of benzo[1,2-b:4,5-b'] diselenophene, intramolecular charge transfer is enhanced, which beneficial to improve $J_{\text {SC. Simultaneously, the weak }}$ intermolecular interaction of F...S, F...Se, and S...O can ameliorate the molecular stacking and morphology to achieve better electron mobility. 


\section{Synthesis}

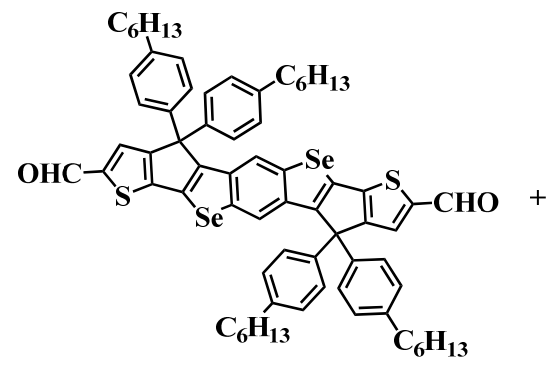

BDSe-CHO<smiles>N#CC(C#N)=C1CC(=O)c2cc(F)c(F)cc21</smiles>

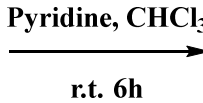

IC-2F

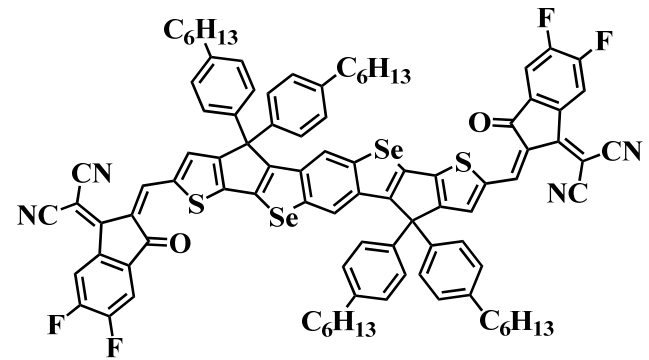

BDSe-4F

Scheme 1. Synthetic route of BDSe-4F

The synthetic route of BDSe-4F was depicted in Scheme 1. The target compound BDSe-4F was obtained by the Knoevenagel condensation reaction between compound BDSe-CHO and compound IC-2F in $88 \%$ yield. In a 100 $\mathrm{mL}$ two-neck round-bottom flask, BDSe-CHO $(0.10 \mathrm{~g}$, $0.08 \mathrm{mmol}), \mathbf{I C}-2 \mathbf{F}(0.11 \mathrm{~g}, 0.48 \mathrm{mmol})$ was added. The reaction mixture was evacuated and backfilled with $\mathrm{N}_{2}$ three times, and then freshly degassed chloroform $(50 \mathrm{~mL})$ and pyridine $(0.5 \mathrm{~mL})$ were added into the reaction mixture. The reaction mixture was stirred at room temperature for $6 \mathrm{~h}$. Then the solution was poured into methanol and the precipitate was filtered off and washed with methanol. The crude product was purified by silica gel column chromatography, eluting with petroleum ether/dichloromethane (1:1) to give the product as purple solid (0.12 g, 88\%). The pure target compound BDSe-4F was characterized by ${ }^{1} \mathrm{H}$ NMR. As shown in Figure 1, ${ }^{1} \mathrm{H}$ $\mathrm{NMR}\left(\mathrm{CDCl}_{3}, 400 \mathrm{MHz}, \mathrm{ppm}\right): \delta 8.84(\mathrm{~s}, 2 \mathrm{H},=\mathrm{CH}), 8.51-$ 8.55 (m, 2H, Ph-H), 8.03 (s, 2H, Th-H), 7.64-7.68 (m, 4H, $\mathrm{Ph}-\mathrm{H}), 7.09-7.16$ (m, $16 \mathrm{H}, \mathrm{Ph}-\mathrm{H}), 2.54-2.58(\mathrm{t}, J=7.6 \mathrm{~Hz}$, $8 \mathrm{H}, \mathrm{CH} 2), 1.55-1.60(\mathrm{~m}, 10 \mathrm{H}, \mathrm{CH} 2), 1.28-1.32(\mathrm{~m}, 22 \mathrm{H}$, $\mathrm{CH} 2), 0.85-0.88$ (m, 12H, CH3)

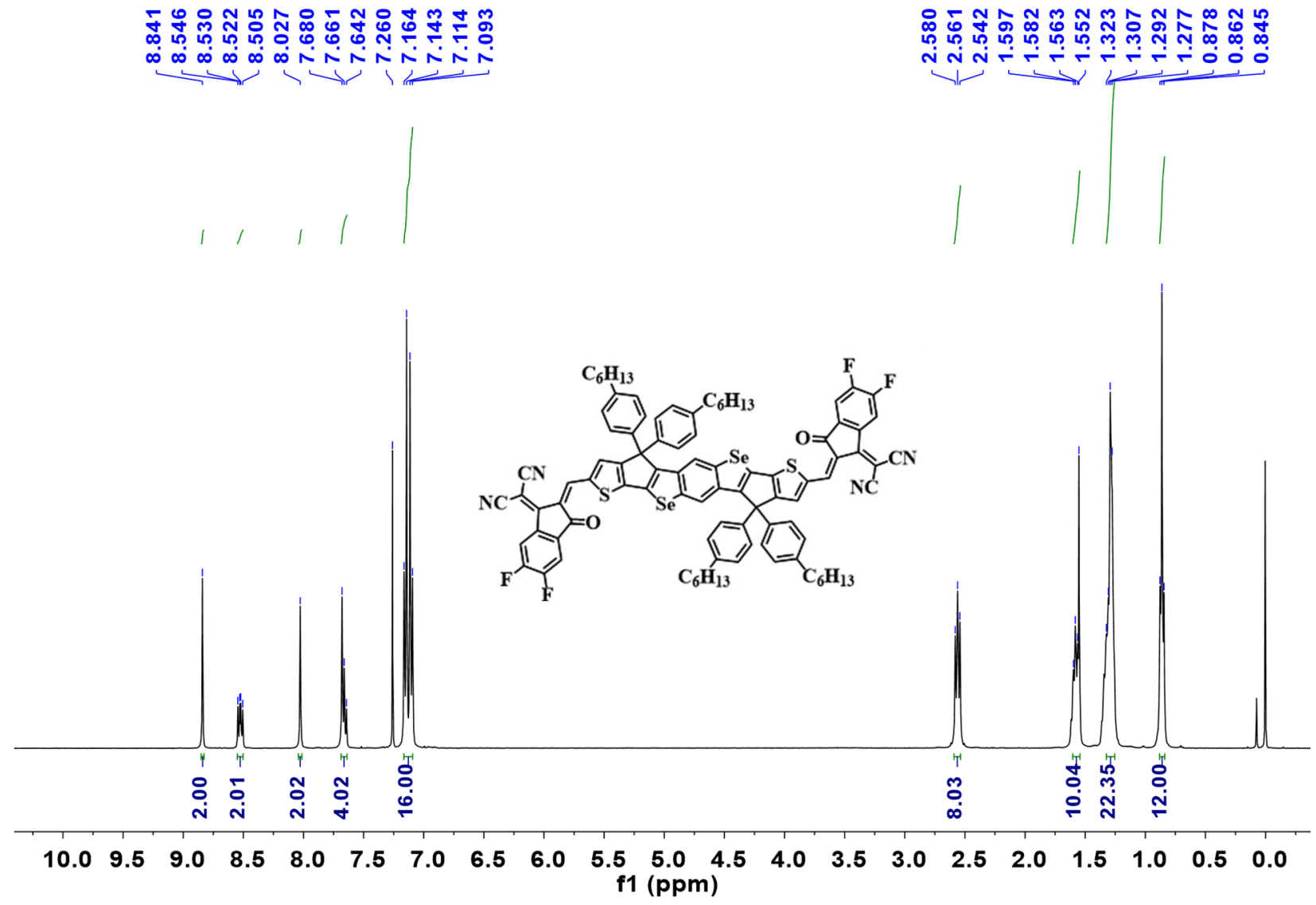

Fig. 1. ${ }^{1} \mathrm{H}$ NMR spectra of BDSe-4F. 


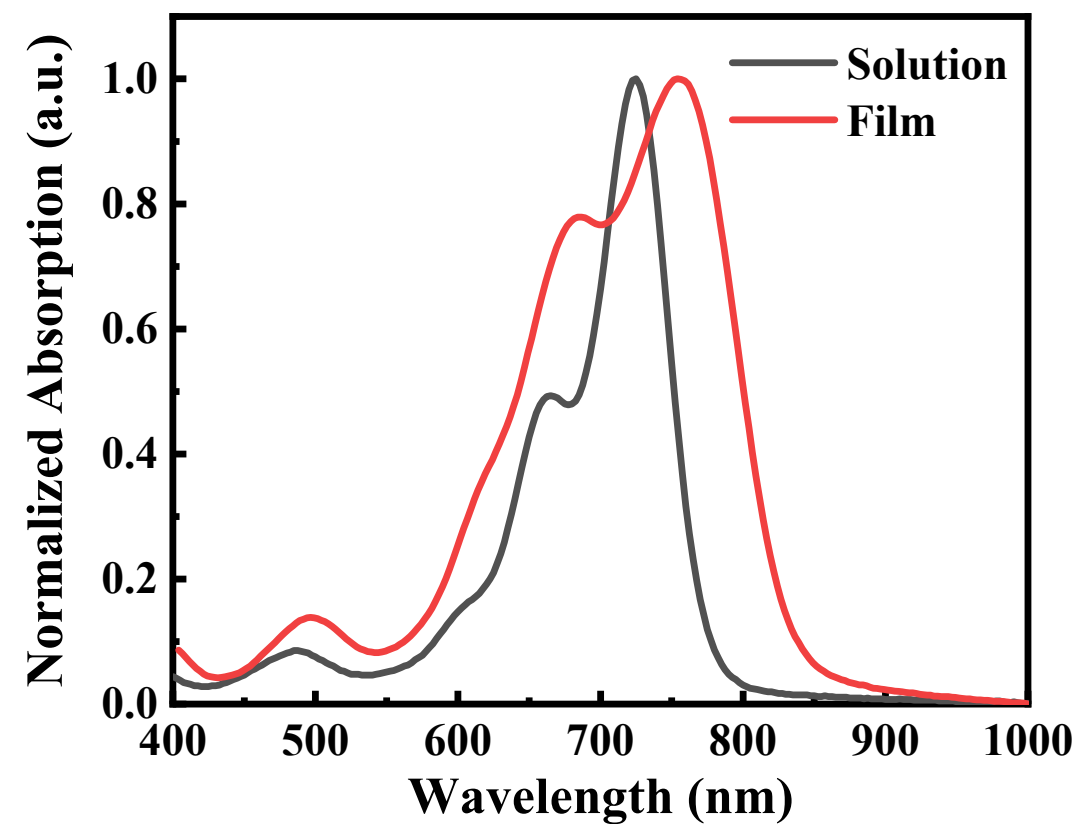

Fig. 2. Normalized absorption spectra of BDSe-4F in chloroform solution and films.

Table 1. Photophysical properties of BDSe-4F in solution and thin films.

\begin{tabular}{ccccc}
\hline Compound & $\begin{array}{c}\lambda_{\max } \text { abs.(sol) } \\
{[\mathrm{nm}]}\end{array}$ & $\begin{array}{c}\lambda_{\max } \begin{array}{c}\lambda_{\text {onset }}(\mathrm{film}) \\
{[\mathrm{nm}]}\end{array} \\
{[\mathrm{nm}]}\end{array}$ & $\begin{array}{c}E_{\mathrm{g}(\mathrm{opt})} \\
{[\mathrm{eV}]}\end{array}$ \\
\hline BDSe-4F & 725 & 756 & 855 & 1.45 \\
\hline
\end{tabular}

\section{Results and discussion}

The normalized absorption spectra of BDSe-4F in dilute chloroform solution and thin film were measured and the relevant parameters are described in Table 1.

As shown in Figure 2, the compound BDSe-4F shows considerable absorption range from $600 \mathrm{~nm}$ to $800 \mathrm{~nm}$ in dilute chloroform solutions. The maximum absorption peaks of BDSe-4F is exhibited at $725 \mathrm{~nm}$ in dilute chloroform solutions. In solid-state films, the maximum absorption peaks of BDSe-4F is exhibited at $756 \mathrm{~nm}$. While for the thin films, the absorption extended broader with an obvious red-shift in the range of $800-900 \mathrm{~nm}$, which attributed to the good intermolecular $\pi-\pi$ stacking in the solid state. In addition, it is important to match with polymer donor on absorption spectrum, which are guideline for choosing donors to pair with NF-SMAs. The compound BDSe-4F better complementary with that of PM6 with the broad cover of the absorption region of 400$800 \mathrm{~nm}$, which is beneficial to capture more photons thus will help increase $J_{\mathrm{SC}}$ in PSCs.

The onset absorption of the compound BDSe-4F shows at $855 \mathrm{~nm}$. Furthermore, the optical bandgap of BDSe-4F was calculated from their absorption edge of the thin film to be $1.45 \mathrm{eV}$. The compound BDSe-4F exhibits a narrower optical bandgap induced by the two fluorine atoms on IC terminal.

\section{Conclusion}

In summary, an A-D-A structure NF-SMA was synthesized and investigated, combined benzo[1,2-b:4,5$\mathrm{b}^{\prime}$ ]diselenophene and the difluorinated 5,6-difluoro-3(dicyanomethylene) indanone (IC-2F). The BDSe-4F shows broad absorption in the range of $600-800 \mathrm{~nm}$ in neat film and exhibits optical band gap of $855 \mathrm{~nm}$. This acceptor is typical narrow band gap NF-SMA. These results indicate that the BDSe-4F can match well with medium band gap polymer donors. In addition, Due to the existence of the $\mathrm{F}$ atom from end groups, the BDSe-4F have weak non-covalent interaction due to F...S, F...Se, and F...S, which beneficial to improve intermolecular $\pi$ $\pi$ stacking and enhance electron mobility. This work demonstrated that the synergistic strategy of end group IC-2F and rich electronic core is a reliable strategy to construct efficient NF-SMAs.

\section{Acknowledgments}

The authors thank Analysis \& Testing Center, Beijing Institute of Technology for NMR experiments. 


\section{References}

1. J. Hou, O. Inganas, R. H. Friend and F. Gao, Nat. Mater., 17, 119-128 (2018).

2. D. Di Carlo Rasi and R. A. J. Janssen, Adv. Mater., 31, 1806499 (2019).

3. P. Cheng, G. Li, X. Zhan and Y. Yang, Nat. Photonics, 12, 131-142 (2018).

4. S. Dai and X. Zhan, Adv. Energy Mater., 8, 1800002 (2018).

5. H. Fu, W. Gao, Y. Li, F. Lin, X. Wu, J. H. Son, J. Luo, H. Y. Woo, Z. Zhu and A. K. Y. Jen, Small Methods, 4, 2000687 (2020).

6. J. Xiong, K. Jin, Y. F. Jiang, J. Q. Qin, T. Wang, J. F. Liu, Q. S. Liu, H. L. Peng, X. F. Li, A. X. Sun, X. Y. Meng, L. X. Zhang, S. W. Shi and L. M. Ding, Sci. Bull., 64, 1573-1576 (2019).

7. Y. Cui, H. Yao, J. Zhang, T. Zhang, Y. Wang, L. Hong, K. Xian, B. Xu, S. Zhang, J. Peng, Z. Wei, F. Gao and J. Hou, Nat. Commun., 10, 2515 (2019).

8. K. Jiang, Q. Y. Wei, J. Y. L. Lai, Z. X. Peng, H. Kim, J. Yuan, L. Ye, H. Ade, Y. P. Zou and H. Yan, Joule, 3, 3020-3033 (2019).

9. Z. H. Luo, R. J. Ma, T. Liu, J. W. Yu, Y. Q. Xiao, R. Sun, G. S. Xie, J. Yuan, Y. Z. Chen, K. Chen, G. D. Chai, H. L. Sun, J. Min, J. Zhang, Y. P. Zou, C. L. Yang, X. H. Lu, F. Gao and H. Yan, Joule, 4, 12361247 (2020).
10. Q. Liu, Y. Jiang, K. Jin, J. Qin, J. Xu, W. Li, J. Xiong, J. Liu, Z. Xiao, K. Sun, S. Yang, X. Zhan and L. M. Ding, Sci. Bull., 65, 272-275 (2020).

11. Y. Cui, H. Yao, J. Zhang, K. Xian, T. Zhang, L. Hong, Y. Wang, Y. Xu, K. Ma, C. An, C. He, Z. Wei, F. Gao and J. H. Hou, Adv. Mater., 32, 1908205 (2020).

12. S. S. Wan, C. Chang, J. L. Wang, Sol. RRL, 3, 1800250 (2019).

13. W. Zhao, S. Li, H. Yao, S. Zhang, Y. Zhang, B. Yang, J. H. Hou, J. Am. Chem. Soc., 139, 7148-7151 (2017).

14. Y. Lin, J. Wang, Z.-G. Zhang, H. Bai, Y. Li, D. Zhu, X. Zhan, Adv. Mater., 27, 1170-1174 (2015).

15. S. Dai, F. Zhao, Q. Zhang, T.-K. Lau, T. Li, K. Liu, Q. Ling, C. Wang, X. Lu, W. You, J. Am. Chem. Soc., 139, 1336-1343 (2017).

16. H. Yao, L. Ye, J. Hou, B. Jang, G. Han, Y. Cui, G. M. Su, C. Wang, B. Gao, R. Yu, Adv. Mater. 29, 1700254 (2017).

17. D. Sun, D. Meng, Y. Cai, B. Fan, Y. Li, W. Jiang, L. Huo, Y. M. Sun, Z. Wang, J. Am. Chem. Soc., 137, 11156-11162 (2015).

18. G. Z. Yuan, H. Fan, J.L. Wang. J. Mater. Chem. A, 7, 20274-20284 (2019).

19. H. Sun, T. Liu, J. Yu, T.-K. Lau, G. Zhang, Y. Zhang, M. Su, Y. Tang, R. Ma, B. Liu, J. Liang, K. Feng, X. Lu, X. Guo, F. Gao and H. Yan, Energy Environ. Sci., 12, 3328-3337, (2019). 